\title{
Coeficiente de Operación de un Sistema formado por un Refrigerador Doméstico Acoplado a un Acumulador de Agua Caliente para la Recuperación de Energía
}

Luís M. P. Souza, Vicente L. Scalon y Alcides Padilha

Universidade Estadual Paulista Júlio de Mesquita Filho, Faculdade de Engenharia de Bauru, Departamento de Engenharia Mecânica, Av. Eng. Luiz Edmundo C. Coube 14-01, CEP 17033-360 Bauru, SP-Brasil (e-mail: luismanoel_tap@hotmail.com, scalon@feb.unesp.br, padilha@feb.unesp.br)

Recibido Jun. 08, 2011; Aceptado Jul. 19, 2011; Versión Final recibida Dic. 27, 2011

\begin{abstract}
Resumen
Se utilizó un aparato experimental formado por un refrigerador doméstico acoplado a un acumulador vertical de agua caliente para la recuperación de energía. El condensador original del refrigerador fue mantenido, pero modificado con un tipo de intercambiador de calor de tubos concéntricos, con el flujo de agua en contracorriente al flujo de gas del refrigerador. El coeficiente de operación de la bomba de calor es calculado por la relación entre la energía captada en el acumulador térmico y la energía eléctrica consumida por el compresor. Los resultados muestran que el incremento de la presión hidrostática en el acumulador térmico aumenta la velocidad de flujo de agua y el coeficiente de operación. El dispositivo diseñado reduce también la disipación de calor para el entorno. Esto es más importante en confinamientos pequeños presentes en las casas de bajo costo.
\end{abstract}

Palabras clave: refrigerador doméstico, almacenamiento térmico, intercambiador de calor, coeficiente de operación

\section{Coefficient of Performance of a System Composed by a Domestic Refrigerator Coupled to a Thermal Storage for Energy Recovery}

\begin{abstract}
An experimental apparatus containing a domestic refrigerator coupled to a vertical hot water storage tank was used for energy recovery. The original condenser of the refrigerator was maintained, but modified with a concentric tubes heat exchanger with countercurrent water and refrigerating gas flows. The coefficient of performance for the heat pump is calculated by the ratio of energy in the heat storage and the electric power consumed by the domestic refrigerator compressor. The results show that the increasing of hydrostatic pressure in the storage tank increases the water flow rate and the coefficient of performance. The proposed device also reduces the heat dissipation to the surroundings. This is more important in small confinements found in low-cost houses.
\end{abstract}

Keywords: domestic refrigerator, thermal storage, heat exchanger, coefficient of performance 


\section{INTRODUCCIÓN}

Con la revolución industrial se incrementó el consumo de energía de recursos naturales no renovables, y con los años este consumo y su dependencia se han intensificado, impulsado principalmente, por las necesidades humanas. Esto se debe a que la sociedad, en general, está pasando por situaciones de crisis con el medio ambiente, que repercuten en la humanidad de diversas maneras, como, por ejemplo, en los desastres ambientales. Teniendo en cuenta esta transformación no controlada, hay un agotamiento de las fuentes de energía tradicionales, intensificando la búsqueda de nuevas fuentes de energías alternativas y renovables. En este contexto, son necesarias investigaciones con el fin de buscar nuevas formas de energía, más eficientes, menos costosas, que sean reciclables, menos degradantes del medio ambiente, y buscar una forma más eficiente para su almacenaje.

Partiendo de lo expuesto, a través de un balance de energía en el sistema de almacenamiento térmico de líquido estratificado, Padilha (1982), propuso un modelo semiempírico unidimensional y transitorio del que se obtuvo el historial de los perfiles de temperaturas del líquido y de la pared del acumulador, durante los períodos de carga, descarga y enfriamiento natural. También se ha comprobado, según la Primera y Segunda Ley de la Termodinámica, la influencia de la relación entre la altura y el diámetro del acumulador en la eficiencia de la estratificación térmica. Se compararon con buena concordancia los resultados teóricos con los resultados experimentales.

Oliveira et al (2008) desarrollaron un modelo matemático para estudiar el comportamiento de la estratificación térmica de líquido en un acumulador para el almacenamiento de energía con medio poroso. Los resultados presentan la influencia de los parámetros físicos de los números de Biot, Darcy, Reynolds, Richardson, la posición y la espesura del medio poroso, para preservación de la estratificación térmica del líquido caliente.

Quinto et al (2005) obtuvieron, experimentalmente, el comportamiento del Coeficiente de Operación de un sistema de refrigeración por compresión mecánica de vapor. Se describe la obtención del Coeficiente de Operación del ciclo de Carnot de refrigeración endorreversible $\left(\mathrm{COP}_{\mathrm{CE}}\right)$, en que se consideran las irreversibilidades externas asociadas a la transferencia de calor. Con los valores de presión y temperatura obtenidos, calcularon el Coeficiente de Operación Real $\left(\mathrm{COP}_{\mathrm{R}}\right)$, el Coeficiente de Operación de Carnot reversible $\left(\mathrm{COP}_{\mathrm{C}}\right)$ y el Coeficiente de Carnot endorreversible $\left(C O P_{C E}\right)$. Al comparar los diferentes valores de los coeficientes de operación, se observó que el $C_{C O P}$ es más cercano al $C_{C O P}$, que el $C_{C O P}$. Esto se confirmó cuando se compararon las relaciones $\left(\mathrm{COP}_{\mathrm{CE}} / \mathrm{COP}_{\mathrm{R}}\right)$, que van de 3.2 a 4.9, con las relaciones $\left(\mathrm{COP}_{\mathrm{C}} / \mathrm{COP}_{\mathrm{R}}\right)$ que van de 14.1 a 32.8. Estos resultados indican que la evaluación de los sistemas de refrigeración es más realista cuando se hace con el COP ${ }_{C E}$ que cuando se hace con el $C O P_{C}$, y se concluye que el $\mathrm{COP}_{\mathrm{R}}$ tiene como valor límite el indicado por el valor del $\mathrm{COP}_{\mathrm{CE}}$.

Luna (2010) analizó numéricamente la existencia de la función potencia de salida y función ecológica, que dan lugar a la forma de las respectivas eficiencias para un ciclo tipo Carnot, llamado ciclo endorreversible, para la máxima potencia de salida y la máxima función ecológica. Se muestra la importancia de dichas funciones para diversos resultados de la literatura relacionada con la termodinámica de tiempos finitos.

Borges et al (2011) analizaron, mediante una simulación numérica, el ciclo de refrigeración de un refrigerador doméstico con el fin de comprobar el consumo de energía eléctrica. Las ecuaciones de conservación de energía, de la conservación de masa y de la cantidad de movimiento, se utilizaron en el modelo matemático, el cual fue validado por los datos experimentales. Los resultados numéricos mostraron una desviación total de $\pm 2 \%$ en comparación con los datos experimentales, mientras que las temperaturas en los compartimientos del refrigerador mostraron una diferencia de $\pm 0,4^{\circ} \mathrm{C}$. El modelo describe cómo la influencia de la apertura de las puertas, las cargas térmicas internas y la descongelación, afectan al consumo de energía.

Amaral Filho et al (2004), construyeron un equipo experimental compuesto de un refrigerador y un acumulador de agua caliente, y desarrollaron un trabajo pionero, en el cual se sustituyó, en su 
totalidad, el condensador del refrigerador por compresión mecánica de vapor, por un intercambiador de calor en contracorriente. Se modificó un refrigerador, colocando un intercambiador en el lugar del condensador, proporcionando un funcionamiento altamente eficiente con flujo de agua en contracorriente al flujo de gas refrigerante. El cambio del condensador por el intercambiador de calor mantuvo el refrigerador modificado funcionando sin alteración en comparación con el refrigerador original. En este sistema, la capacidad de almacenamiento de agua caliente es de 85 litros, no siendo aplicados los coeficientes de operaciones irreversible, reversible o endorreversible, para calcular la eficiencia del sistema integrado: bomba de calor y acumulador de energía.

Con parte del equipo de Amaral Filho et al (2004), pero con un acumulador de 122 litros, en su trabajo experimental, de Marchi Neto et al (2009), obtuvieron y compararon el Coeficiente de Operación Experimental $\left(\mathrm{COP}_{\mathrm{EXP}}\right)$, por la relación entre la energía térmica en el acumulador térmico y la energía eléctrica consumida por el compresor de cada uno de los refrigeradores, uno convencional y otro modificado. En el refrigerador convencional el coeficiente fue obtenido considerando las pérdidas térmicas, a través de las paredes del refrigerador y del aire en los compartimientos refrigerados con la energía eléctrica consumida por el compresor. El refrigerador modificado, tuvo también su condensador original sustituido por un intercambiador de calor con el flujo de agua en contracorriente al gas refrigerante. En este caso, el coeficiente fue calculado considerando la cuantía de calor absorbido por el agua en el intercambiador y almacenado para uso doméstico, en relación a la energía eléctrica consumida por el compresor. El refrigerador con el condensador modificado fue más eficiente en comparación con el sistema convencional, reduciendo el consumo de energía eléctrica en los compresores. Los resultados mostraron que en el refrigerador modificado no hay difusión de calor en el medio ambiente, ni tampoco en las paredes de los compartimentos del refrigerador a enfriar. La cuestión a ser planteada, en el caso del refrigerador modificado, es que, para el acumulador completamente cargado de agua caliente, el gas no se condensa y el refrigerador cesa su operación.

Tanto en los estudios experimentales de de Marchi Neto et al (2009) como los de Amaral Filho et al (2004), el condensador original del refrigerador fue sustituido en su totalidad por un intercambiador de calor enfriado por agua en contracorriente con la velocidad de flujo de gas refrigerante. Aunque hay un incremento en la eficiencia del refrigerador, este incremento funcionará mientras que el agua caliente no llegue al fondo del depósito y, por lo tanto, al intercambiador de calor, ya que si esto ocurre, el refrigerador dejará de funcionar. Con ello, será necesario un estudio para descubrir el punto de equilibrio entre la oferta y la demanda de agua caliente, o una alteración del sistema, sin cambiar las condiciones originales de funcionamiento del refrigerador.

Teniendo en cuenta lo expuesto anteriormente, los objetivos de este trabajo son: partir de la utilización de parte del aparato experimental que fue construido por de Marchi Neto et al (2009), es decir, el tanque de almacenamiento de 122 litros de agua caliente y la misma altura de 0,70 m, entre la salida de agua caliente del intercambiador de calor y la entrada de la misma en la parte superior del tanque, para mantener o aumentar la eficacia del refrigerador. En este trabajo experimental se mantuvo el condensador original en el que se añadió un intercambiador de calor a la parte superior del referido condensador de aletas, manteniendo el funcionamiento del refrigerador en el caso del tanque completamente lleno de agua caliente. De esta forma, se obtuvo el Coeficiente de Operación Experimental $\left(\mathrm{COP}_{\mathrm{EXP}}\right)$ de la energía en el acumulador térmico, con relación a la energía eléctrica consumida por el compresor, para dos casos de presión hidrostática en el sistema, lo cual produce un aumento en el flujo de agua a través del sistema. Se analizó, también, el almacenamiento por estratificación térmica de agua caliente y se calcularon las velocidades de flujos de agua y de gas refrigerante que circulan a través del sistema.

\section{MATERIALES, MÉTODOS Y MODELO MATEMÁTICO}

El aparato experimental es muestrado en la Fig.1, consiste en parte de un equipo construido por de Marchi Neto et al (2009), es decir, el tanque de almacenamiento de 122 litros de agua caliente (1,75 metros de altura y 0,3 metros de diámetro) y altura de $0,70 \mathrm{~m}$, entre la salida de agua 
caliente del intercambiador de calor y la entrada de la misma en la parte superior del acumulador térmico. En este trabajo experimental se utilizo un refrigerador doméstico, con un compartimiento de refrigeración con capacidad de 263 litros, un compartimiento de congelación de 74 litros, se mantuvo el condensador original en el que se añadió un intercambiador de calor a la parte superior del referido condensador de aletas y un sistema de adquisición de datos. La energía eléctrica consumida por el compresor del refrigerador, se midió a través de un registrador electrónico de magnitudes eléctricas para sistemas monofásicos. Se utilizó la misma altura admitido por de Marchi Neto et al (2009) en su proyecto experimental, o sea, 0,70 metros.
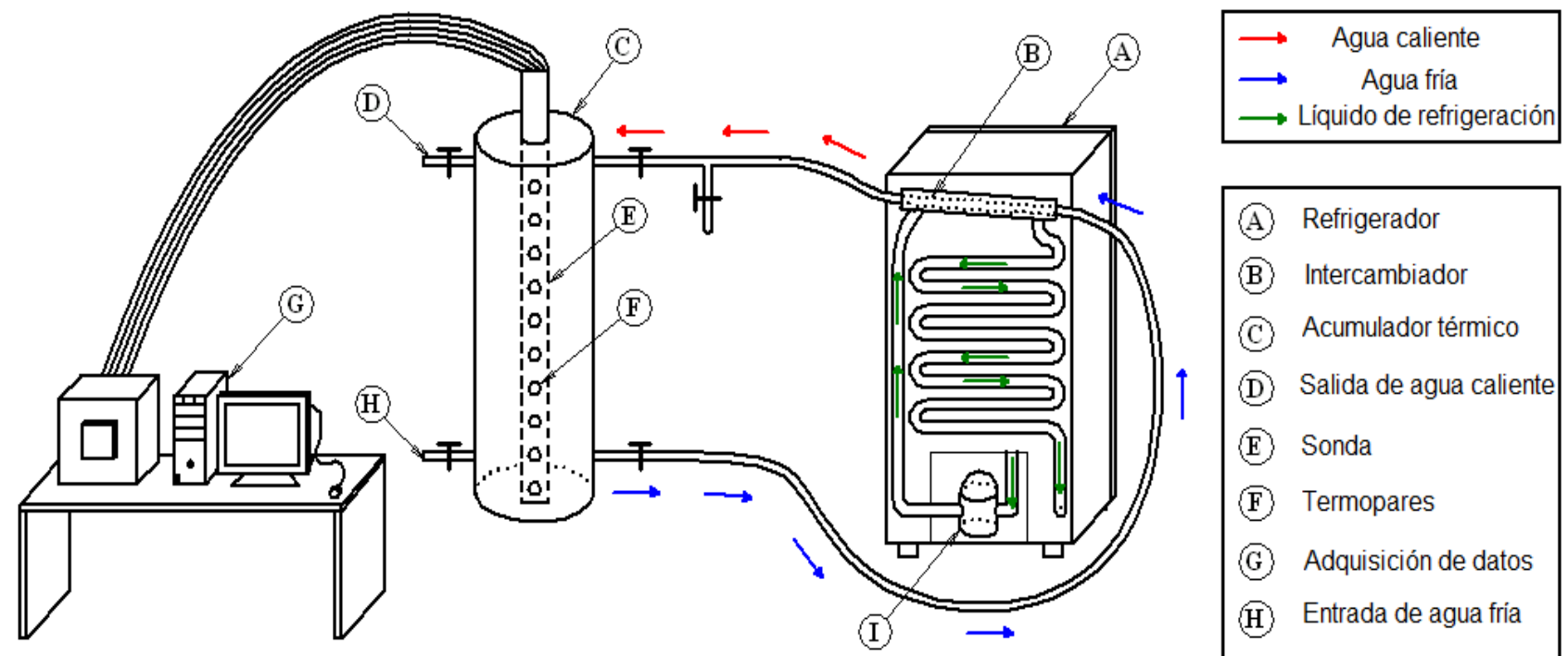

(A) Refrigerador
(B) Intercambiador
(C) Acumulador térmico
(D) Salida de agua caliente
(E) Sonda
(F) Termopares
(G) Adquisición de datos
(H) Entrada de agua fría
(I) Compresor

Fig. 1: Esquema general de la instalación experimental.

La dinámica de la carga del acumulador se realiza mediante la inyección de agua caliente desde el intercambiador de calor, por la parte superior del tanque, seguido de la extracción del mismo flujo de agua fría por la parte inferior del acumulador, en dirección al intercambiador de calor. De este modo, se estableció el proceso de estratificación térmica del agua, es decir, la formación de tres zonas térmicas en el interior del acumulador: una caliente, una fría y una intermedia, llamada isóclina, entre las dos primeras zonas. Al estudiar el movimiento del frente cálido, obtenido a través del proceso de estratificación térmica, se determina el tiempo de recorrido de éste, entre los termopares dispuestos equidistantes de $0,05 \mathrm{~m}$, en una sonda en el interior del acumulador térmico. De esta forma, en función de la geometría del acumulador, se determinará la velocidad del flujo de agua caliente que circula a través del sistema, para dos valores de presiones hidrostáticas, sin y con columna de agua de altura máxima de 0,10 metros, medido en la escala de un tubo transparente fijo en la parte superior del acumulador térmico.

\section{DETERMINACIÓN DE COEFICIENTE DE OPERACIÓN EXPERIMENTAL (COPEXP)}

De la Ec. 1 se calcula la velocidad de flujo volumétrico del agua caliente en función de la geometría del tanque y, el tiempo $(t)$ se calcula por el desplazamiento del frente cálido entre dos termopares consecutivos.

$\dot{V}=\frac{\pi \times r^{2} \times \Delta L}{t}$

A través de la velocidad del flujo de agua caliente y de los niveles de temperatura y presión, medidos en la entrada y salida del intercambiador de calor, se obtiene (indirectamente) la velocidad del flujo del gas refrigerante (R-134a), circulando por el refrigerador, suponiendo que 
haya una igualdad entre el flujo de calor intercambiado entre el agua y el gas refrigerante, o sea, no hay pérdidas de calor al ambiente en el intercambiador de calor. Experimentalmente el agua no recupera totalmente el calor del gas refrigerante y así menos calor almacenado en el acumulador, disminuyendo la eficiencia del sistema.

$$
\dot{Q}_{\text {gas }}=Q_{\text {agua }}
$$

Por lo tanto,

$$
\left[\dot{m}\left(h_{\text {salida }}-h_{\text {entrada }}\right)\right]_{\text {gas }}=\left[\dot{m} \times c_{p} \times\left(T_{\text {salida }}-T_{\text {entrada }}\right)\right]_{\text {gqua }}
$$

De esta forma:

$$
[\dot{m}]_{\text {gas }}=\frac{\dot{m} \times c_{p} \times\left(T_{\text {salida }}-T_{\text {entrada }}\right)_{\text {agua }}}{\left(h_{\text {salida }}-h_{\text {entrada }}\right)}
$$

La cantidad de calor almacenado en cada capa de fluido en el acumulador se calculó mediante la relación:

$$
Q_{n}=\left[m_{\text {agua }} \times c_{p_{\text {agua }}} \times\left(T m_{f}-T m_{i}\right)\right]_{C n}
$$

Donde $f$ e $i$ representan los instantes final e inicial.

El índice suscrito $C n$ es relativo a las capas del fluido almacenado, y varía entre $1 \leq n \leq 30$, ya que hay 31 termopares en la sonda para obtener cálculos más precisos de la energía almacenada, aunque en la Fig. 3 se muestra sólo cuatro perfiles de temperatura.

La cantidad total de calor almacenado en el acumulador térmico entre los instantes inicial y final se expresa mediante la Ec. 6, es decir:

$$
Q_{T}=\sum_{n=1}^{30}\left[m_{\text {agua }} \times c_{p_{\text {agua }}} \times\left(T m_{f}-T m_{i}\right)\right]_{C n}
$$

Al generalizar en todos los instantes de tiempo, tendremos:

$$
Q_{T}=\sum_{i=1}^{t_{\text {aq }}} \sum_{n=1}^{30}\left[m_{\text {agua }} \times c_{p_{\text {agua }}} \times\left(T m_{f}-T m_{i}\right)\right]_{C n}
$$

Donde $t_{a q}$ es el tiempo transcurrido entre dos adquisiciones de datos.

Una vez calculado el $Q_{T}$ (de Marchi Neto et al (2009)) se determina el coeficiente de operación del sistema acumulado $\left(\mathrm{COP}_{\mathrm{EXP}}\right)$, relacionando la cantidad de energía almacenada $Q_{T}$ con la energía eléctrica consumida por el compresor $W$, que corresponde al coeficiente de operación experimental de una bomba de calor:

$$
\operatorname{COP}_{E X P}=\frac{Q_{T}}{W}
$$




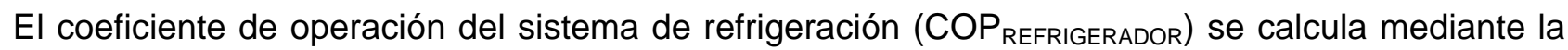
relación entre el calor extraído del compartimiento del refrigerador y la energía eléctrica consumida por el compresor.

\section{RESULTADOS}

En la Fig. 2 se muestra la velocidad del flujo de agua caliente según las temperaturas de la salida del intercambiador de calor. Se puede observar una disminución de las velocidades de los flujos de agua con el aumento de la temperatura en la salida del referido intercambiador. La disminución en la velocidad del flujo ocurrió debido a la reducción de la carga térmica con el tiempo en los compartimentos de refrigeración, lo que resulta en menos calor que se intercambia entre el gas refrigerante y el agua.

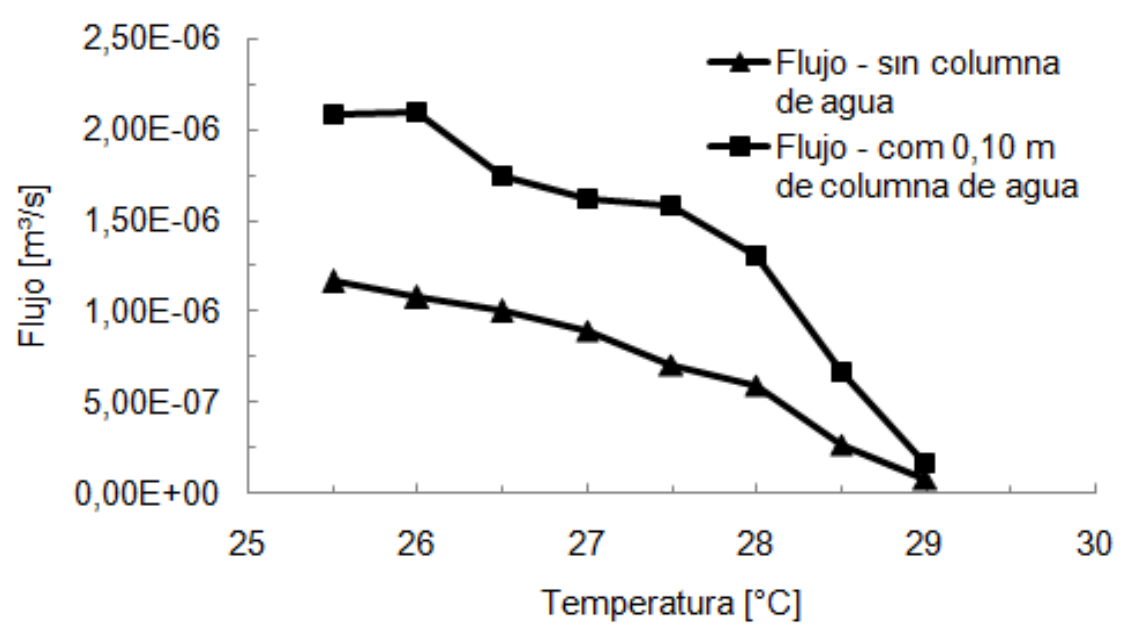

Fig. 2: Velocidad de flujo del agua caliente en función de la temperatura en la salida del intercambiador de calor.

En la Tabla 1 se muestra la velocidad de los flujos medios de agua caliente y del gas, la energía eléctrica consumida, la energía térmica almacenada, medidas experimentalmente, y el $\mathrm{COP}_{\mathrm{EXP}}$ con relación a la presión del agua en el depósito del acumulador térmico.

En la Tabla 1, se puede observar que la energía eléctrica consumida es mayor que la energía térmica almacenada, es decir, los coeficientes no son superiores a la unidad, esto es debido a las pérdidas de calor en algunas partes del equipo, como, por ejemplo, en el compresor, intercambiador y en el acumulador. También hay un aumento en el $\mathrm{COP}_{\mathrm{EXP}}$ a medida que aumenta la presión del agua en el depósito y, por lo tanto, mayor caudal de agua caliente en la salida del intercambiador. Este aumento del COP $\operatorname{EXP}_{\text {se }}$ debe a una mayor eficiencia de intercambio de calor entre el gas refrigerante y el agua en el intercambiador.

Tabla 1: Velocidad de los flujos medios de agua caliente y del gas, energía eléctrica consumida, energía térmica almacenada y el $\mathrm{COP}_{\mathrm{EXP}}$ con relación a la presión del agua en el depósito del acumulador térmico.

\begin{tabular}{|c|c|c|c|c|c|}
\hline $\begin{array}{c}\text { Columna de } \\
\text { agua } \\
{[\mathrm{m}]}\end{array}$ & $\begin{array}{c}\text { Velocidad de flujos } \\
\text { medios de agua } \\
\text { caliente }[\mathrm{l} / \mathrm{h}]\end{array}$ & $\begin{array}{c}\text { Flujos de gas } \\
\text { refrigerante } \\
{[\mathrm{l} / \mathrm{h}]}\end{array}$ & $\begin{array}{c}\text { Energía eléctrica } \\
\text { consumida } \\
{[\mathrm{MJ}]}\end{array}$ & $\begin{array}{c}\text { Energía } \\
\text { térmica } \\
\text { almacenada } \\
{[\mathrm{MJ}]}\end{array}$ & COP $_{\text {EXP }}$ \\
\hline 0 & 3,78 & 4,8 & 7,2 & 2,0 & 0,27 \\
\hline 0,10 & 5,82 & 5 & 7,2 & 2,9 & 0,40 \\
\hline
\end{tabular}

En la Fig. 3 se presenta el proceso de estratificación térmica del agua en el interior del acumulador por un período de prueba de 24 horas, con una velocidad de flujo de agua de $5,82 \mathrm{l} / \mathrm{h}$. Con el aumento del tiempo, se nota el inicio del proceso de estratificación térmica, lo que demuestra la 
formación de la zona caliente. En función del tiempo, se destaca la formación de tres zonas distintas de temperaturas distribuidas a lo largo del acumulador: una zona caliente en la parte superior, una zona fría en el fondo, y una zona intermedia entre las dos anteriores. Hay que tener en cuenta, también, la superposición de los perfiles de temperatura, resultantes de la cantidad de energía almacenada, la cual no es suficiente para que ocurra una estratificación térmica uniforme.

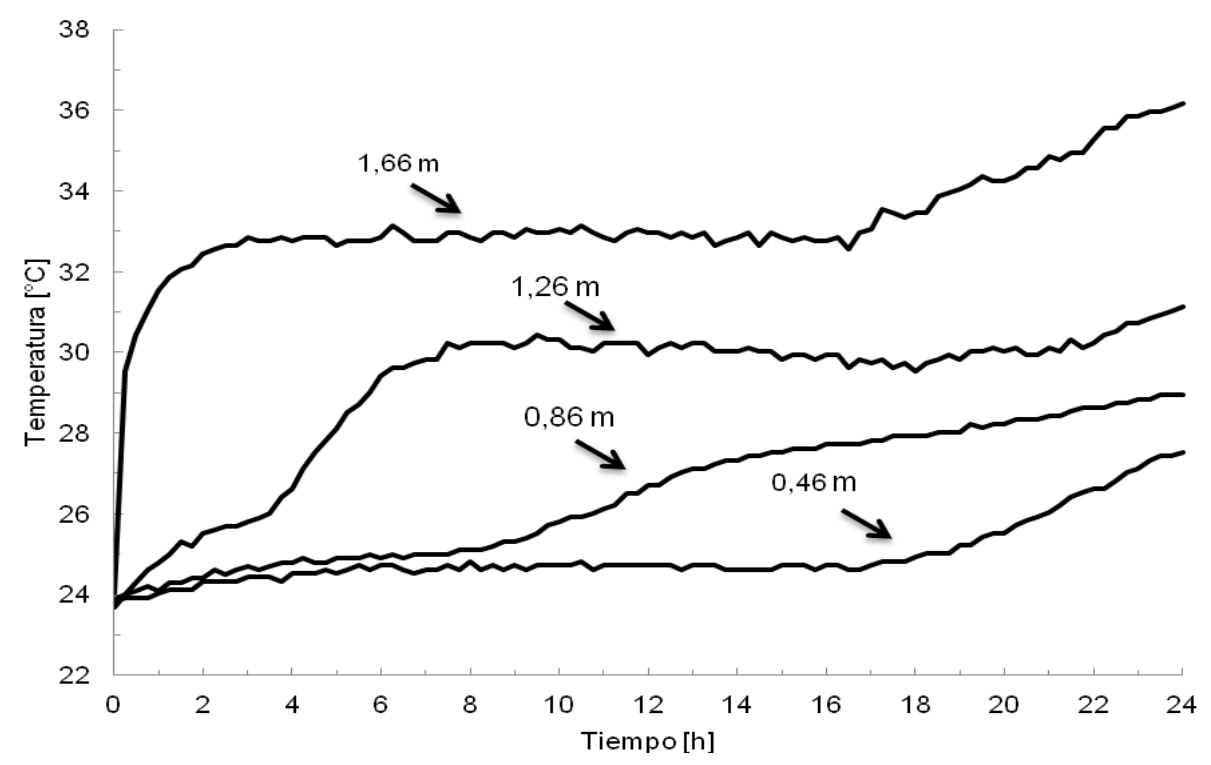

Fig. 3: Evolución de los perfiles de temperatura con respecto a la posición de cada termopar en el interior del acumulador térmico.

La Fig. 4 muestra el comportamiento de las isóclinas a lo largo de la altura del acumulador, a cada ocho horas de pruebas, teniendo en cuenta el tiempo de carga de 24 horas y la velocidad del flujo de agua caliente de $5,82 \mathrm{l} / \mathrm{h}$.

Se observa la deformación de la isóclina inicial $(\mathrm{t}=0 \mathrm{~h})$ con el tiempo, lo cual simula la dinámica de carga de agua caliente en el acumulador. La disposición de las isóclinas con relación a la primera $(t=0 \mathrm{~h}$ ) es el resultado de la cantidad de calor almacenado que aumenta con el tiempo. Se observa que no se produce una cantidad uniforme de energía acumulada con el tiempo, debido a la disminución de la carga térmica del refrigerador, con el paso del tiempo.

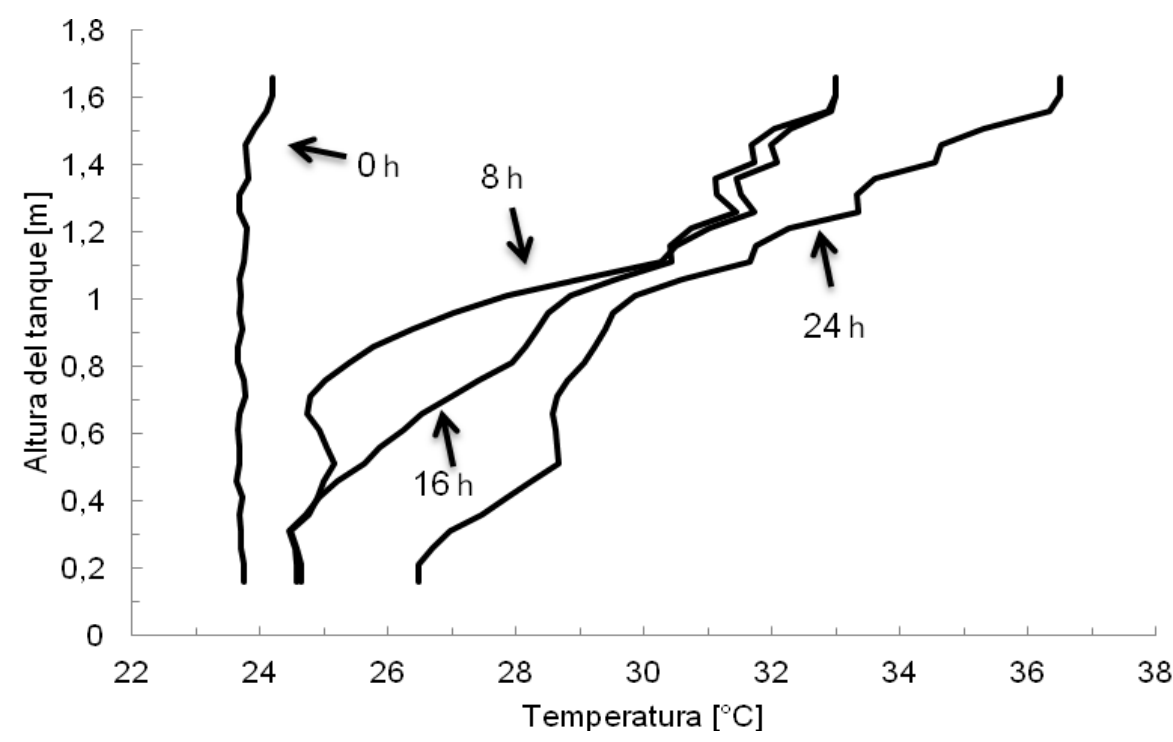

Fig. 4: Comportamiento de las isóclinas a lo largo de la altura del tanque. 
La Fig. 5 muestra el aumento de consumo de energía eléctrica a través del compresor con el tiempo. Este aumento de consumo es independiente de la variación de la presión del agua en el depósito. Se puede observar, también, una pequeña variación de la energía térmica almacenada en forma de calor sin y con columna de agua. La figura indica que es posible una mejora en el sistema de captación de energía, ya que gran parte de ésta no fue recuperada por el dispositivo del sistema experimental, pues, teóricamente, la cantidad de calor almacenado tiene que ser mayor que la cantidad de energía inyectada al sistema a través del compresor. Con estos datos se calcula el coeficiente de operación del sistema acumulado (COP EXP), como se ilustra en la Tabla 1.

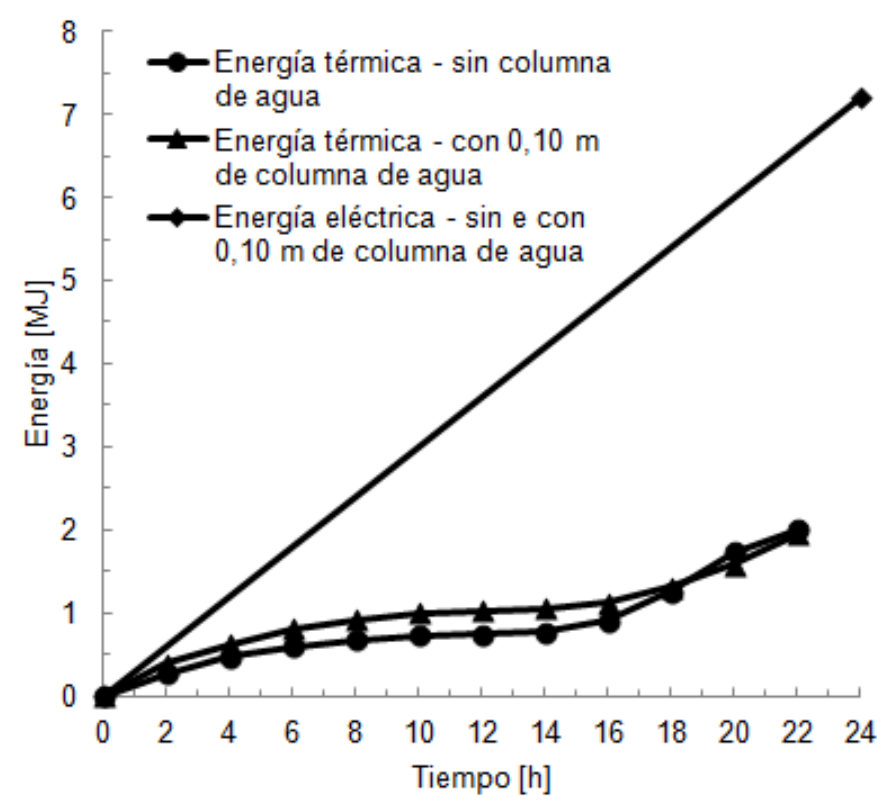

Fig. 5 muestra la energía eléctrica consumida por el compresor, y la energía térmica acumulada en el acumulador en los dos experimentos.

En la Fig. 6, se muestran los perfiles de los $\mathrm{COP}_{\mathrm{EXP}}$ en función del tiempo. Se observa que, con el paso del tiempo, el $\mathrm{COP}_{\mathrm{EXP}}$ tiende a cero, debido al desplazamiento del frente de agua caliente hacia la región inferior del acumulador, lo que resulta en la entrada de agua caliente en el intercambiador, sin embargo, el condensador original inicia su cambio de calor con el gas refrigerante y el refrigerador no cesa su operación.

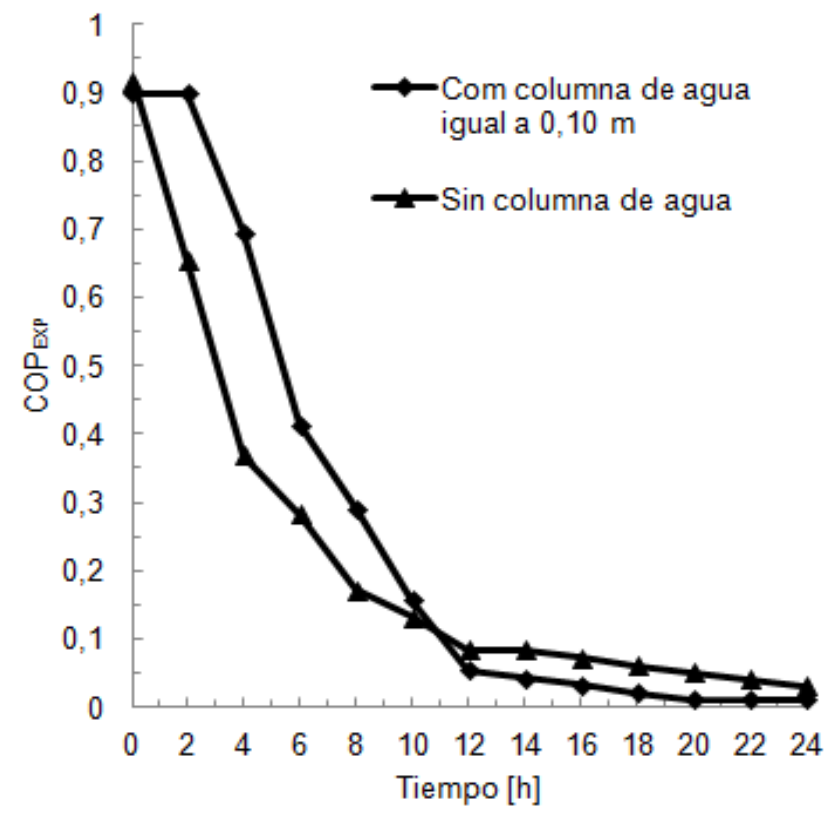

Fig. 6: $\mathrm{COP}_{\mathrm{EXP}}$ en función del tiempo para los dos experimentos analizados. 


\section{CONCLUSIONES}

Según los resultados obtenidos en este trabajo, se puede concluir que:

Con el paso del tiempo, el $\mathrm{COP}_{\operatorname{EXP}}$ (Fig. 6) tiende a cero, debido al desplazamiento del frente de agua caliente hacia la región inferior del acumulador, lo que resulta en la entrada de agua caliente en el intercambiador. Cuando esto ocurre, el condensador original inicia su cambio de calor con el gas refrigerante y el refrigerador no cesa su operación, lo que no ocurre con la propuesta de de Marchi Neto et al (2009).

En el caso del refrigerador modificado, con el condensador original, el sistema continúa produciendo agua caliente para uso doméstico; y con el aumento de la presión hidrostática del acumulador térmico, ocurre un aumento de la velocidad del flujo de agua caliente y, por lo tanto, un aumento del COP EXP , lo que se puede observar en la Fig. 6 hasta 11 horas.

Para que funcione este sistema experimental de recuperación de calor del refrigerador, aún es necesario aumentar la recuperación de la energía almacenada para obtener valores del coeficiente superior a la unidad. Por lo tanto, es necesario reducir las pérdidas de calor en algunas partes del equipo, como, por ejemplo, el compresor y el acumulador. Este proceso está, actualmente, en desarrollo.

\section{AGRADECIMIENTOS}

Los autores agradecen a la CAPES por el apoyo financiero.

\section{NOMENCLATURA}

$\mathrm{COP}_{\mathrm{EXP}}$ Coeficiente de operación experimental del sistema refrigerador / acumulador

$c_{p} \quad$ Calor específico a presión constante (J/kg.K)

$C_{n} \quad$ Referente a las capas de fluido almacenado

$h \quad$ Entalpía $(\mathrm{kJ} / \mathrm{kg})$

$\Delta L \quad$ Espaciamiento entre los termopares de la sonda $(\mathrm{m})$

i $\quad$ Velocidad del flujo de masa $(\mathrm{kg} / \mathrm{s})$

$n \quad$ Referente al número de termopares

$\dot{Q} \quad$ Flujo de calor (W)

$Q_{T} \quad$ Cantidad total de energía térmica almacenada (J)

$r \quad$ Radio del acumulador térmico $(\mathrm{m})$

$T \quad$ Temperatura $\left({ }^{\circ} \mathrm{C}\right)$

Tm Temperatura media $\left({ }^{\circ} \mathrm{C}\right)$

$t \quad$ Tiempo [s]

$t_{a q} \quad$ Referente al tiempo de adquisición de datos

$\dot{V} \quad$ Velocidad del flujo volumétrico $\left(\mathrm{m}^{3} / \mathrm{s}\right)$

W Energía eléctrica consumida por el refrigerador (J)

subíndices

i Instante inicial

$f \quad$ Instante final

aq adquisición

$\mathrm{T}$ total

$\mathrm{n} \quad$ número de capas de fluido almacenado 


\section{REFERENCIAS}

Amaral Filho, P. H. F., Scalon, V., Padilha, A, Análise Teórica e Experimental da Captação e Utilização do Calor Dissipado no Condensador de Sistemas de Refrigeração, $10^{\circ}$ Brasilian Congress of Thermal Engineering and Sciences - Encit, Rio de Janeiro, (2004).

Borges, B. N., Hermes, C. J.L., Gonçalves, J.M., Melo, C, Transiente simulation of household refrigerators: A semi-empirical quasi-steady approach, Applied Energy: 88, 748-754 (2011).

de Marchi Neto, I., Scalon, V., Padilha, A, Refrigerator COP with thermal storage, Applied Thermal Engineering: 29, 2358-2364 (2009).

Luna, D. I., Análisis del Comportamiento de un Ciclo de Tipo Carnot, Información tecnológica: 21, 4, 79-86 (2010).

Oliveira, S., del R., Padilha, A., Scalon, V., L., Analysis of the Accumulated Energy by Thermal Stratification of Liquid Inside Storage Tanks With Porous Layers, Numerical Heat Transfer, Part A, 53: 377-391, (2008).

Padilha, A, Estocagem por estratificação térmica de líquido em reservatório, I Congresso Latino Americano de Transferência de Calor e Massa, Argentina: 2, 934-949 (1982).

Quinto, P., Carvajal, I., Sánchez, F., Abugaber, J, Evaluación de un sistema de refrigeración usando la termodinámica endorreversible, Información tecnológica: 16, 5, 49-55 (2005). 